Mathematical Research Letters 3, 723-729 (1996)

\title{
GENERALIZED LIOUVILLE PROPERTIES OF MANIFOLDS
}

\author{
Tobias H. Colding and William P. Minicozzi II
}

In this paper, we announce results on generalized Liouville theorems on manifolds.

Our first theorem is the following.

Theorem 1. [CM4] For an open manifold with nonnegative Ricci curvature the space of harmonic functions with polynomial growth of a fixed rate is finite dimensional.

We show Theorem 1 by giving an explicit bound on the dimension of this space depending only on the dimension of the manifold and the rate of growth.

Theorem 1 was conjectured by Yau (see [Y3], [Y4], [Y5], and [L1]). Around $1974 \mathrm{Yau}$, [Y1], generalized the classical Liouville theorem of complex analysis to open manifolds with nonnegative Ricci curvature. Specifically, he proved that a positive harmonic function on such a manifold must be constant. Yau's Liouville theorem was considerably generalized by Cheng-Yau (see $[\mathrm{CgY}]$ ) by means of a gradient estimate which implies the Harnack inequality. As a consequence of this gradient estimate (see $[\mathrm{Cg}]$ ), on such a manifold nonconstant harmonic functions must grow at least linearly.

The case $n=2$ of Theorem 1 was done earlier by Peter Li and L.F. Tam, [LT2] (in fact, for surfaces with finite total curvature). For another proof in the case $n=2$ using nodal sets see Harold Donnelly and Charles Fefferman, [DF]. In [LT1] Peter Li and L.F. Tam settled the case $d=1$. In our earlier paper [CM2] (see also [CM1]), we proved the above conjecture of Yau under the additional assumption that $M^{n}$ has Euclidean volume growth. We refer to the references for additional results related to this conjecture.

We recall the definition of polynomial growth.

\footnotetext{
Received June 17, 1996.

The first author was partially supported by NSF Grant DMS-9504994 and an Alfred P. Sloan Research Fellowship and the second author by an NSF Postdoctoral Fellowship.
} 
Definition. For an open (complete noncompact) manifold, $M^{n}$, given a point $p \in M$ let $r$ be the distance from $p$. Define $\mathcal{H}_{d}(M)$ to be the linear space of harmonic functions with order of growth at most $d$. This means that $u \in \mathcal{H}_{d}$ if $u$ is harmonic and there exists some $C<\infty$ so that $|u| \leq C\left(1+r^{d}\right)$.

In fact, Theorem 1 is a consequence of a much more general result. In order to state this we need to recall the definition of some basic analytic inequalities on Riemannian manifolds.

Let $\left(M^{n}, g\right)$ be a complete Riemannian manifold.

Doubling property. We say that $M^{n}$ has the doubling property if there exists $C_{D}<\infty$ such that for all $p \in M^{n}$ and $r>0$

$$
\operatorname{Vol}\left(B_{2 r}(p)\right) \leq C_{D} \operatorname{Vol}\left(B_{r}(p)\right) .
$$

Neumann Poincaré inequality. We say that $M^{n}$ satisfies an uniform Neumann Poincaré inequality if there exists $C_{N}<\infty$ such that for all $p \in M^{n}, r>0$ and $f \in W_{l o c}^{2,1}(M)$

$$
\int_{B_{r}(p)}(f-\mathcal{A})^{2} \leq C_{N} r^{2} \int_{B_{r}(p)}|\nabla f|^{2},
$$

where $\mathcal{A}=\frac{1}{\operatorname{Vol}\left(B_{r}(p)\right)} \int_{B_{r}(p)} f$.

Note that if $M^{n}$ is a manifold with nonnegative Ricci curvature, then $M^{n}$ has the doubling property with doubling constant $C_{D}=2^{n}$ by the classical relative volume comparison theorem. Observe also that in $[\mathrm{Bu}]$ Peter Buser showed that these manifolds satisfy an uniform Neumann Poincaré inequality with $C_{N}=C_{N}(n)<\infty$.

We can now state our more general result.

Theorem 2. [CM4] If $M^{n}$ is an open manifold which has the doubling property and satisfies an uniform Neumann Poincaré inequality, then for all $d>0$, there exists $C=C\left(d, C_{D}, C_{N}\right)<\infty$ such that $\operatorname{dim} \mathcal{H}_{d}(M) \leq C$.

We note that a particular consequence of our proof is that we need very little regularity of the metric.

An immediate consequence of Theorem 2, in addition to Theorem 1, is the following.

Corollary 3. [CM4] If $M^{n}$ is an open manifold which is quasi isometric to an open manifold with nonnegative Ricci curvature then $\operatorname{dim} \mathcal{H}_{d}(M)<$ $\infty$ for all $d>0$. 
Recall that two metric spaces are said to be quasi isometric if they are bilipschitz.

In [Ly] Lyons gave examples showing that, in general, Liouville properties are not stable under quasi isometric changes. However, Saloff-Coste, [Sa1], and Grigor'yan, [Gr], independently showed that if an open manifold has nonnegative Ricci curvature then any other quasi isometric manifold does not admit any nonconstant bounded harmonic function. In fact, Saloff-Coste, [Sa2], and Grigor'yan, [Gr], gave a lower bound in terms of $n, C_{D}$ and $C_{N}$ for the rate of growth of a nonconstant harmonic function on a manifold which has an uniform Neumann Poincaré inequality and has the doubling property.

Prior to the results of [Gr], [Sa1], [Sa2], Lyons and Sullivan, [LySu], and Guivarc'h, $[\mathrm{Gu}]$, showed that a normal cover with nilpotent deck group of a closed manifold does not admit a nonconstant bounded (or more generally positive) harmonic function. As a generalization of this we have the following immediate consequence of Theorem 2 (see for instance [Gv] for the definition of polynomial growth of a finitely generated group).

Corollary 4. [CM4] Suppose that $M^{n}$ is a closed manifold and $\bar{M}$ is a normal cover of $M$ with deck group of polynomial growth. For all $d>0$, $\operatorname{dim} \mathcal{H}_{d}(\bar{M})<\infty$.

For elliptic operators in divergence form on $\mathbb{R}^{n}$ we get the following theorem.

Theorem 5. [CM4] If $L$ is a quasi uniformly elliptic operator in divergence form on $\mathbb{R}^{n}$ then the linear space of L-harmonic functions on $\mathbb{R}^{n}$ with polynomial growth of a fixed rate is finite dimensional.

We refer to [CM4] for the exact definitions involved in the statement of Theorem 5. Here we will only note that quasi uniformly elliptic is more general than uniformly elliptic.

For an area minimizing hypersurface $\Sigma^{n} \subset \mathbb{R}^{n+1}$, we define the spaces $\mathcal{H}_{d}(\Sigma)$ with respect to the Euclidean distance. We have the following immediate application of Theorem 2 .

Theorem 6. [CM4] Let $\Sigma^{n} \subset \mathbb{R}^{n+1}$ be a complete area minimizing hypersurface without boundary, $L$ an uniformly elliptic divergence form operator on $\Sigma$, and $d>0$. If $\Sigma$ has Euclidean volume growth, then $\operatorname{dim} \mathcal{H}_{d}(\Sigma, L)<$ $\infty$

In [CM5] we obtain Weyl type asymptotic bounds for the dimension of the space of harmonic functions of polynomial growth on manifolds of 
nonnegative Ricci curvature. Namely we prove the following more general theorem (in fact this theorem holds even for quasi uniformly elliptic operators on these manifolds).

Theorem 7. [CM5] Suppose that $M^{n}$ has an uniform Neumann Poincaré inequality and has the doubling property then there exist $C=C\left(C_{D}, C_{N}\right)<\infty$ such that for $d>0$

$$
\operatorname{dim} \mathcal{H}_{d}\left(M^{n}\right) \leq C d^{\frac{\log C_{D}}{\log 2}}
$$

As an application, we obtain a Siegel's theorem bounding the transcendence degree of the quotient field associated to the algebra of polynomial growth holomorphic functions on an open Kähler manifold which is quasi isometric to a manifold with nonnegative Ricci curvature.

In [CM6], we will give a generalization of Theorem 6 and some geometric applications of this.

Theorem 8. [CM6] Let $\Sigma^{n} \subset \mathbb{R}^{n+k}$ be a complete minimally immersed submanifold without boundary which has Euclidean volume growth with $\operatorname{Vol}\left(\Sigma \cap B_{r}(x)\right) \leq V r^{n}$ for all $r>0$ and all $x \in \mathbb{R}^{n+k}$. For any $d>0$, $\operatorname{dim} \mathcal{H}_{d}(\Sigma) \leq C$ where $C=C(n, V, d)<\infty$.

There are many examples of minimal submanifolds satisfying the hypotheses of Theorem 8; for instance, any affine algebraic submanifold of $\mathbb{C}^{N}$.

Since the coordinate functions are themselves in $\mathcal{H}_{1}(\Sigma)$, we get the following Bernstein type corollary of Theorem 8.

Corollary 9. [CM6] If $\Sigma^{n} \subset \mathbb{R}^{n+k}$ is a complete minimally immersed submanifold without boundary which has Euclidean volume growth with $\operatorname{Vol}\left(\Sigma \cap B_{r}(x)\right) \leq \operatorname{Vr}^{n}$ for all $r>0$ and all $x \in \mathbb{R}^{n+k}$, then $\Sigma$ must be contained in some affine subspace of dimension at most $\ell=\ell(n, V)$.

We note that Corollary 9 was previously proven for minimal submanifolds of spheres, and hence for minimal cones in Euclidean space, by ChengLi-Yau, $[\mathrm{CgLY}]$, using heat equation methods.

Also in [CM6], we prove Liouville type theorems for harmonic sections of bundles with nonnegative curvature on manifolds with nonnegative Ricci curvature. For a vector bundle $E \rightarrow M$ with a hermitian metric and $d>0$, define the space $\mathcal{H}_{d}(M, E)$ as an obvious generalization of the definition of $\mathcal{H}_{d}$ for functions.

Theorem 10. [CM6] Let $M^{n}$ be an open manifold with nonnegative Ricci curvature and $E^{k}$ a rank $k$ hermitian vector bundle with nonnegative curvature. For all $d>0, \operatorname{dim} \mathcal{H}_{d}(M, E)<\infty$. 
In fact, we show Theorem 10 by giving an explicit bound on the dimension of $\mathcal{H}_{d}(M, E)$ depending only on $n, d$, and $k$. As a corollary of Theorem 10, we get the following result which addresses a question raised by Yau, [Y4] and [Y5].

Corollary 11. [CM6] Let $M^{n}$ be a Kähler manifold with nonnegative Ricci curvature and $E$ a hermitian holomorphic line bundle with nonnegative curvature. For all $d>0, \operatorname{dim} \mathcal{H}_{d}(M, E)<\infty$.

See $[\mathrm{CM} 5]$ and $[\mathrm{CM} 6]$ for additional results in this direction.

\section{References}

[BCr] R. Bishop and R. Crittenden, Geometry of manifolds, Academic Press (1964).

[BoG] E. Bombieri and E. Giusti, Harnack's inequality for elliptic differential equations on minimal surfaces, Invent. Math. 15 (1972), 24-46.

[Bu] P. Buser, A note on the isoperimetric constant, Ann. Scient. Ec. Norm. Sup. 15 (1982), 213-230.

[ChC1] J. Cheeger and T. H. Colding, Lower bounds on the Ricci curvature and the almost rigidity of warped products, Ann. of Math. 144 (1996), 189-237.

[ChC2] _ _ On the structure of spaces with Ricci curvature bounded below; I, J. Diff. Geom., (to appear).

[ChC3] _ _ Almost rigidity of warped products and the structure of spaces with Ricci curvature bounded below, C.R. Acad. Sci. Paris t. 320 (1995), 353-357.

[ChCM] J. Cheeger, T. H. Colding, and W. P. Minicozzi II, Linear growth harmonic functions on complete manifolds with nonnegative Ricci curvature, Geom. Funct. Anal. 5 (1995), 948-954.

[Cg] S. Y. Cheng, Liouville theorem for harmonic maps, Proc. Symp. Pure Math. 36 (1980), 147-151.

[CgLY] S. Y. Cheng, P. Li, and S.-T. Yau, Heat equations on minimal submanifolds and their geometric applications, Amer. J. Math. 106 (1984), 1033-1065.

[CgY] S. Y. Cheng and S.-T. Yau, Differential equations on Riemannian manifolds and their geometric applications, Comm. Pure Appl. Math. 28 (1975), 333354.

[CnZ] T. Christiansen and M. Zworski, Harmonic functions of polynomial growth on certain complete manifolds, Geom. Funct. Anal. 6 (1996), 619-627.

[C1] T. H. Colding, Ricci curvature and volume convergence, Ann. of Math., (to appear).

[C2] _ _ Stability and Ricci curvature, C.R. Acad. Sci. Paris t. 320 (1995), 1343$\overline{1347 .}$.

[CM1] T. H. Colding and W. P. Minicozzi II, On function theory on spaces with a lower Ricci curvature bound, Math. Res. Lett. 3 (1996), 241-246. 
[CM2] _ Harmonic functions with polynomial growth, J. Diff. Geom., (to appear).

[CM3] _ _ Large scale behavior of kernels of Schrödinger operators, preprint.

[CM4] _ Harmonic functions on manifolds, Ann. of Math., (to appear).

[CM5] _ Weyl type bounds for harmonic functions, preprint.

[CM6] Liouville theorems for harmonic sections and applications, (to appear).

[DF] H. Donnelly and C. Fefferman, Nodal domains and growth of harmonic functions on noncompact manifolds, J. Geom. Anal. 2 (1992), 79-93.

[Gr] A. A. Grigor'yan, The heat equation on noncompact Riemannian manifolds, English trans. in Math. USSR Sb. 72 (1992), 47-77.

[Gv] M. Gromov, Groups of polynomial growth and expanding maps, Publ. IHES 53 (1982), 53-78.

[Gu] Y. Guivarc'h, Mouvement brownien sur les revêtements d'une variété compacte, C.R. Acad. Sci. Paris 292 (1981), 851-853.

[K1] A. Kasue, Harmonic functions of polynomial growth on complete manifolds, Proc. Sympos. Pure Math. 54 Amer. Math. Soc. (1993), 281-290.

[K2] _ Harmonic functions of polynomial growth on complete manifolds II, J. Math. Soc. Japan 47 (1995), 37-65.

$[\mathrm{Kz}]$ J. Kazdan, Parabolicity and the Liouville property on complete Riemannian manifolds, Aspects of Mathematics E10 Braunschwieg:Vieweg (1987), 153166.

[L1] P. Li, The theory of harmonic functions and its relation to geometry, Proc. Sympos. Pure Math. 54 Amer. Math. Soc. (1993), 307-315.

[L2] _ Linear growth harmonic functions on Kähler manifolds with nonnegative Ricci curvature, Math. Res. Lett. 2 (1995), 79-94.

[LT1] P. Li and L.-F. Tam, Linear growth harmonic functions on a complete manifold, J. Diff. Geom. 29 (1989), 421-425.

[LT2] _ Complete surfaces with finite total curvature, J. Diff. Geom. 33 (1991), 139-168.

[Ln] F. H. Lin, Asymptotically conic elliptic operators and Liouville type theorems, preprint.

[Ly] T. Lyons, Instability of the Liouville property for quasi-isometric Riemannian manifolds and reversible Markov chains, J. Diff. Geom. (1987) 33-66.

[LySu] T. Lyons and D. Sullivan, Function theory random paths and covering spaces, J. Diff. Geom. 19 (1984), 299-323.

[Sa1] L. Saloff-Coste, Uniformly elliptic operators on Riemannian manifolds, J. Diff. Geom. 36 (1992), 417-450.

[Sa2] _ A note on Poincaré, Sobolev, and Harnack inequalities, Int. Math. Res. Not. 2 (1992), 27-38.

[SY] R. M. Schoen and S.-T. Yau, Lectures on differential geometry, International Press, Cambridge, MA, 1995.

[W] J. Wang, Linear growth harmonic functions on complete manifolds, Comm. Anal. Geom. 3 (1995), 683-698.

[Wu] H. Wu, Polynomial functions on complete Kähler manifolds, Proc. Sympos. Pure Math. 52 Amer. Math. Soc. (1991), 601-610.

[Y1] S.-T. Yau, Harmonic functions on complete Riemannian manifolds, Comm. Pure and Appl. Math. 28 (1975), 201-228.

[Y2] _ _ Some function-theoretic properties of complete Riemannian manifold and their applications to geometry, Indiana Univ. Math. Journal 25 (1976), 659-670. 
[Y3] _ Nonlinear analysis in geometry, L'Eseignement Mathematique 2 (1987), 109-158.

[Y4] _ Open problems in geometry, Proc. Symp. Pure Math. 54 Ed. R. Greene and S.-T. Yau.

[Y5] __ Open problems in geometry, Chern - A Great Geometer of the Twentieth Century, Ed. S.T. Yau, International Press, Cambridge, MA, 1992.

Courant Institute of Mathematical Sciences, 251 Mercer St., New York, NY 10012

E-mail address: colding@cims.nyu.edu

Department of Mathematics, Johns Hopkins University, 3400 N. Charles St., BALTIMORE, MD 21218

E-mail address: minicozz@math.jhu.edu 